\title{
Antihypertensive Medication Exposure and Cardiovascular Outcomes in Hemodialysis Patients
}

\author{
Theresa I. Shireman ${ }^{a, b}$ Milind A. Phadnis ${ }^{c}$ James B. Wetmore ${ }^{d}$ \\ Xinhua Zhou $^{\mathrm{e}}$ Sally K. Rigler ${ }^{f}$ John A. Spertus ${ }^{g}$, h Edward F. Ellerbeck $^{\mathrm{a}}$ \\ Jonathan D. Mahnken ${ }^{c}$ \\ ${ }^{a}$ Department of Preventive Medicine and Public Health, ${ }^{\mathrm{b}}$ The Kidney Institute, and ${ }^{\mathrm{c}}$ Department of Biostatistics, \\ University of Kansas School of Medicine, Kansas City, Kans., ' Hennepin County Medical Center, Minneapolis, Minn.,

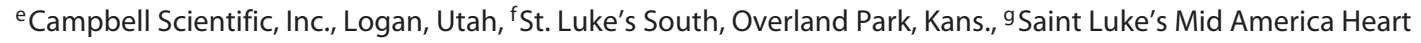 \\ Institute, and h University of Missouri-Kansas City, Kansas City, Mo., USA
}

\section{Key Words}

Antihypertensive drugs · Outcomes · Dialysis · End-stage

renal disease $\cdot$ Medicare $\cdot$ Medicaid

\begin{abstract}
Background/Aims: Our understanding of the effectiveness of cardioprotective medications in maintenance dialysis patients is based upon drug exposures assessed at a single point in time. We employed a novel, time-dependent approach to modeling medication use over time to examine outcomes in a large national cohort. Methods: We linked Medicaid prescription claims with United States Renal Data System registry data and Medicare claims for 52,922 hypertensive maintenance dialysis patients. All-cause mortality and a combined cardiovascular disease (CVD)-endpoint were modeled as functions of exposure to cardioprotective antihypertensive medications (renin angiotensin system antagonists, $\beta$-adrenergic blockers, and calcium channel blockers) measured with three time-dependent covariates (weekly exposure status, proportion of prior weeks with exposure,
\end{abstract}

and number of switches in exposure status) and with propensity adjustment. Results: Current cardioprotective medication exposure status as compared to not exposed was associated with lower adjusted hazard ratios (AHRs) for mortality, though the magnitude depended upon the proportion of prior weeks with medication (duration) and the number of switches between active and non-active use (switches) (AHR range 0.54-0.90). Combined CVD-endpoints depended upon the proportion of weeks on medication: $\mathrm{AHR}=1.18$ for $10 \%$ and $A H R=0.90$ for $90 \%$ of weeks. Combined CVDendpoint was also lower for patients with fewer switches. Conclusions: Effectiveness depends not only on having a drug available but is tempered by duration and stability of use, likely reflecting variation in clinical stability and patient behavior.

(c) 2014 S. Karger AG, Basel

Preliminary results were presented at the American Society of $\mathrm{Ne}$ phrology Kidney Week Meeting, November 2012, San Diego, Calif., USA.

\section{KARGER}

E-Mail karger@karger.com www.karger.com/ajn
(C) 2014 S. Karger AG, Basel

0250-8095/14/0402-0113\$39.50/0
Theresa I. Shireman, $\mathrm{PhD}$

3901 Rainbow Blvd, MS 1008

Kansas City, KS 66160 (USA)

E-Mail tshireman@kumc.edu 


\section{Background/Aims}

Hypertension and cardiovascular diseases are common in end-stage renal disease (ESRD) patients on dialysis and have an important adverse impact on morbidity and mortality $[1,2]$. Despite a strong clinical rationale to preferentially prescribe antihypertensive agents with cardioprotective properties (renin angiotensin system antagonists, $\beta$-adrenergic blockers, and calcium channel blockers) in this population, the benefits of these agents have only rigorously been shown in the non-dialysis population [3-15]. Persons on maintenance dialysis have been excluded from nearly all large-scale clinical trials [5, 16-18], although a handful of trials that have been conducted in maintenance dialysis patients suggest some level of efficacy, though in the presence of publication bias $[19,20]$. The publication of a recent randomized clinical trial comparing atenolol to lisinopril patients on maintenance dialysis with low ejection fraction signals continued need for clinical understanding in this area [21].

Previous observational studies examining the effectiveness of these medications in maintenance dialysis cohorts [22-36] have a number of shortcomings [22]. Most did not adjust for non-random treatment assignment. Additionally, medication use was documented at a single point in time with potentially several years of follow-up, failing to account for actual drug exposure over time. Given the clinical instability of dialysis patients, it is likely that many subjects experience a more variable pattern of medication exposure over time.

In order to better model the complexity of medication exposure over time, we developed a novel set of time-dependent medication exposure metrics that captured contemporaneous use, cumulative use up to that point in time, and the number of changes in exposure status to date. We hypothesized that use of medications from these three classes would be associated with reduced all-cause mortality and cardiovascular endpoints. However, we also hypothesized that those associations would be tempered by each of three aforementioned dimensions of longitudinal exposure, with stronger effects seen for those with higher continuous exposure, fewer switches, and remaining actively on the medications.

\section{Methods}

\section{Study Design}

We conducted a retrospective cohort study of incident maintenance dialysis patients with hypertension, tracking their longitudinal exposure to cardioprotective antihypertensive medica- tions [e.g. angiotensin-converting enzyme inhibitors (ACEIs) and angiotensin receptor blockers (ARBs), $\beta$-adrenergic blockers ( $\beta$-blockers), and calcium channel blockers (CCBs)] and assessing their outcomes for up to 6 years (2000-2005). Outcomes were allcause mortality (ACM) and a combined cardiovascular disease endpoint (CVD-endpoint). We applied both propensity score adjustment and time-dependent modeling of medication exposure to determine the effectiveness of ACEIs/ARBs, $\beta$-blockers, and $\mathrm{CCBs}$ in reducing outcome events in patients on maintenance dialysis.

\section{Participants}

The cohort consisted of newly dialyzing, adult ( $\geq 18$ years), dually-eligible (Medicaid and Medicare) subjects matched against the United State Renal Data System (USRDS). Medicaid is a state-federal program that provides healthcare insurance for persons with low incomes in the United States: Medicare is a federal program that provides healthcare insurance for persons with ESRD and older adults in the United States. We used the dually eligible so as to track prescription medication exposure provided through Medicaid coverage (outpatient prescription medications were not covered under Medicare during this period). The USRDS, a national registry of maintenance dialysis patients, matched Medicaid beneficiaries against their core files to identify dual eligibles on maintenance dialysis and provided us a common linking variable across datasets [37].

To assure complete observation of cohort, we instituted several criteria. First, we limited the study population to persons enrolled in a single state's Medicaid, fee-for-service program. Patients with coverage through the Veterans Administration and subjects who had previously been transplanted but were returning to maintenance dialysis were excluded. Persons who had received a transplant, died, or were not continuously eligible for Medicare and Medicaid during the first 90 days on dialysis were excluded. Persons who did not fill any prescriptions during the first 90 days were excluded; this lack of prescriptions was thought to reflect the Medicaid's spend-down requirements. Ohio residents were excluded since their claims do not include the days supplied of medication. We also excluded persons who were institutionalized during their entire follow-up period, were missing multiple data fields from their CMS 2728, and/or did not have hypertension documented on the CMS 2728.

The observation window began immediately following a 90 day run-in period. We considered the 91 st day after initiating dialysis as the first day of observation in our cohort. Subsequently, subjects were followed until they incurred a first outcome event (death or combined CVD-endpoint) and were censored at the time they lost Medicare or Medicaid eligibility, were transplanted, or reached the end of the observation window (12/31/2005).

\section{Data Sources}

We obtained data from the USRDS and the Centers for Medicare and Medicaid Services (CMS). From CMS, we obtained Medicaid Analytic eXtract (MAX) Personal Summary Files as well as the final action prescription drug claims files (2000-2005). The USRDS provided several files including their Core CD with baseline registry data collected from the CMS 2728 Medical Evidence Form along with data on payer status mortality, and Medicare Parts A (hospital) and B (physician) claims. 


\section{Covariate Measures}

The USRDS Core CD included information documented upon initiation of maintenance dialysis through the CMS 2728 Medical Evidence Form. From this form, we ascertained baseline demographic and behavioral risk variables: age, sex, and race by ethnicity, employment, smoking and substance abuse, functional status (ability to ambulate and ability to transfer), body mass index (BMI), and major comorbidities. We also used a measure of disease burden, called the modified Liu Comorbidity Index in development of the propensity score [38]: this index combines baseline comorbidities from the CMS 2728 with diagnosis codes recorded in Medicare Part A and Part B claims and has been validated in the context of survival prediction with similar performance to the Charlson score [38]. We modified Liu's comorbidity score by reducing the claims period from 270 to 90 days so as to identify an expanded set of baseline conditions (cerebrovascular accident, peripheral vascular disease, other cardiac disease, chronic obstructive pulmonary disease, gastrointestinal disease, liver disease, cancer, and heart failure) [37]. The presence of individual conditions was weighted as outlined in the original scoring [38]. Finally, baseline dialysis modality was categorized as in-center hemodialysis (HD) or home dialysis [home HD or peritoneal dialysis (PD)] at initiation.

\section{Medication Exposure}

We characterized medication exposure from Medicaid prescription drug claims which detail the National Drug Code (NDC), the date filled, and day's supply of medication dispensed across the window of observation. We linked NDC codes to individual $\beta$-blockers, ACEI/ARBs, and CCBs whether in single or combined products (drug names available upon request). The longitudinal exposure measures involved taking each date a study medication was dispensed (prescription claim) and creating a data array for day-by-day availability after the date of dispensing based upon day's supply. We adjusted for early refills ( $>1$ week from expected date for refill), outlier values, and hospital and skilled nursing facility days, as medications are acquired and administered through the institutional service and would not result in an outpatient drug claim. We repeated this process characterizing exposure to any drug from one of the three classes, as prescribers often switch from one class to another. The combined measure captures exposure to a $\beta$-blocker, ACEI/ARB, or CCB during this day-byday data array. As a means of validating our exposure-outcome models, we also included levothyroxine exposure as an 'inert' medication unlikely to improve survival or cardiovascular outcomes [39].

We subsequently modeled medication exposure with three time-dependent variables tracked on a weekly basis: exposure status (on/off) during the week based on at least 1 day in the week with medication, proportion of preceding weeks with drug exposure, and the cumulative number of switches between exposed and unexposed status (on-to-off or off-to-on) across the preceding weeks.

\section{Outcomes}

ACM was ascertained from the USRDS Core CD, which specifies the date and cause of death. In addition, we created a combined CVD-endpoint, capturing the first event per person. Cardiovascular-related morbidity was defined as an inpatient hospitalization (Medicare Part A claims) for myocardial infarction (International
Classification of Diseases Version 9, ICD9, codes: 410.x0, 410.x1), ischemic heart disease (411.xx), revascularization (ICD9 procedure codes 36.xx except 36.9), congestive heart failure (CHF) (428. $\mathrm{xx}, 402 . x 1,404 . x 1$, or 404.x3), cerebrovascular accident (433.xx, 434.xx, 435.x), or peripheral vascular disease (PVD) (440.2-4, 443.1, 443.81, 443.9, 444.2x, 444.81, 445.0x). Cardiac-related mortality was derived from the USRDS listed cause of death (myocardial infarction, atherosclerotic heart disease, cardiomyopathy, cardiac arrhythmia, cardiac arrest, cerebrovascular accidents) [27]. Outcome events were quantified as to occurrence and time to event or censoring.

\section{Statistical Analyses}

We first adjusted for propensity to be a user for the combined drug class exposure. Propensity scores were derived using predicted probabilities from unconditional logistic regression models using baseline CMS 2728 measures (age, sex, and race by ethnicity, employment, smoking and substance abuse, functional status, BMI) and the modified Liu comorbidity index. Propensity models initially included main effects only, and based on the HosmerLemeshow lack-of-fit test, were modified to include all possible higher-order interactions until the lack-of-fit test was no longer rejected. The propensity score was then used as an adjustor in subsequent models.

To investigate effectiveness, we fit time-dependent Cox regression models to identify separately the covariates (including the propensity score) associated with mortality and combined CVDendpoint. A priori, interactions between these three time-dependent measures were assumed to reflect the pathway of mortality or morbidity benefits conferred by medication usage, if any. Specifically, this allowed us the potential to evaluate overall medication effectiveness as a function of varying medication usage patterns between patients. To evaluate the statistical significance of such interactions, we started with a full three-way interaction model between these time-dependent measures subsequently reducing the models to their most parsimonious analogs. Exponentiation of the parameter estimates obtained from these models using appropriate contrast statements allowed us to calculate the hazard ratios for evaluating medication effectiveness as a function of these timedependent measures.

For covariates other than those associated with medication exposure (non-time-dependent), Cox proportionality assumptions were ascertained using standard goodness-of-fit tests. Statistical significance was inferred when $\mathrm{p}<0.01$. All statistical analyses were done with SAS 9.2 (SAS Institute, Inc., www.sas. com). We present results for the combined drug classes for the time-dependent models in the paper: individual classes results are in an attached supplement. The parameter estimates from the individual drug classes were quite similar to the combined analysis.

\section{Compliance and Research Participant Protection}

The research protocol was approved by the institutional review board at the University of Kansas Medical Center (KUMC). Data Use Agreements between KUMC and the USRDS and CMS permitted the data linking across the USRDS, Medicare and Medicaid files. 
Table 1. Descriptive characteristics of the incident, Medicare-Medicaid eligible maintenance dialysis patients with hypertension initiating dialysis January 2000-October 2005

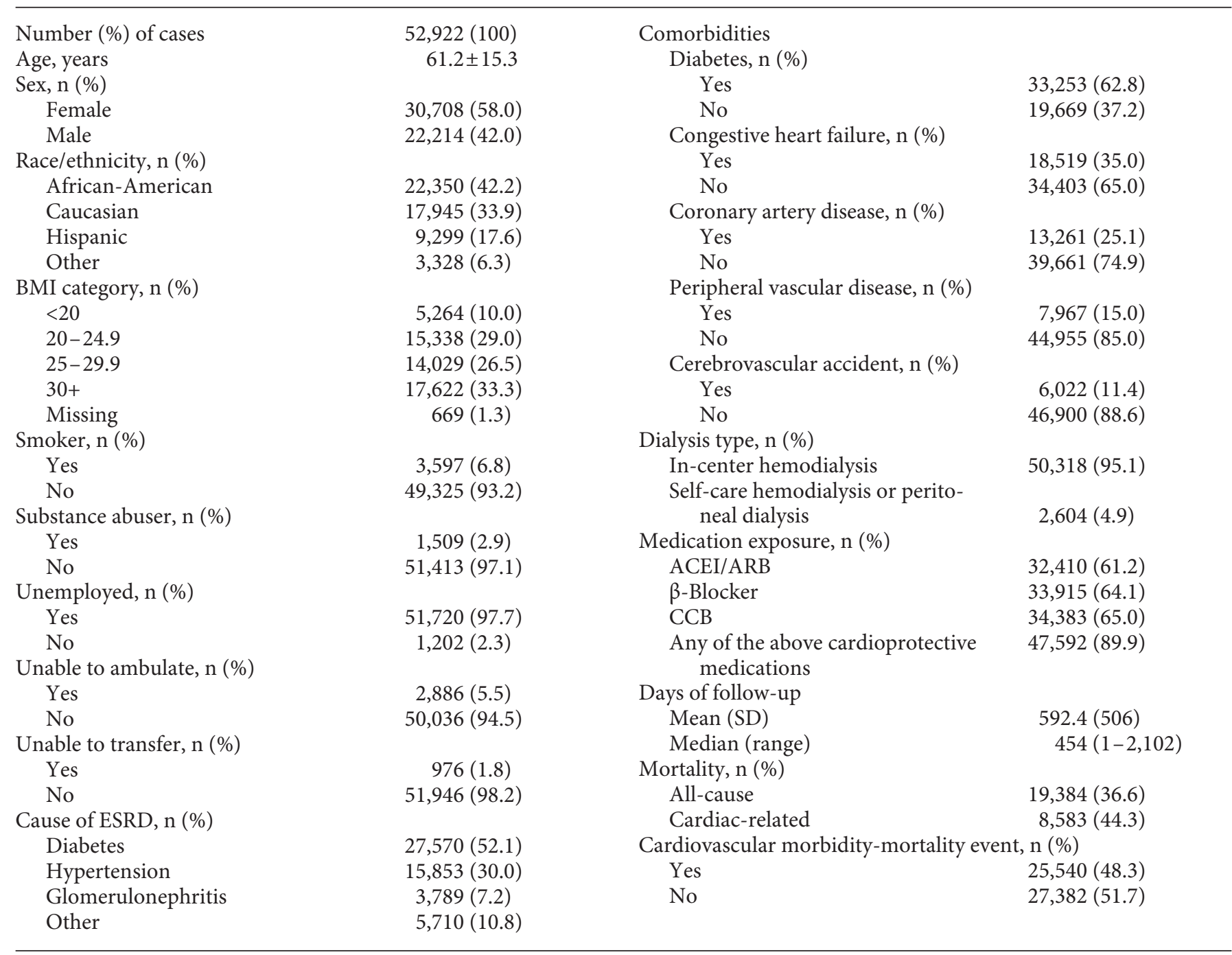

\section{Results}

\section{Characteristics of the Analytic Cohort}

Steps in the data linking and cohort construction resulted in 52,922 incident maintenance dialysis patients with hypertension (online suppl. fig. S1; see www. karger.com/doi/10.1159/000365255 for all online suppl. material). The cohort had an average age of $61.2 \pm 15.3$ years and included more females (58.0\%) than males (table 1). African-Americans were the predominant racial/ ethnic group (42.2\%), followed by Caucasians (33.9\%), and Hispanics (17.6\%). Only $2.3 \%$ were employed. Just over half of the cohort (52.1\%) had diabetes as the primary cause for renal failure, followed by hypertension
(30.0\%). CHF was present in $35.0 \%$, coronary artery disease (CAD) in $25.1 \%$, and $11.4 \%$ had a history of a CVA. Most received in-center HD. The final cohort was quite comparable to the initial dually eligible, observable cohort $(\mathrm{n}=84,670)$ across baseline characteristics (table available upon request).

The majority of the cohort (89.9\%) was exposed to at least one of the cardioprotective medications (table 1). Rates for the individual classes were $61.2 \%$ for ACEI/ ARB, $64.1 \%$ for $\beta$-blockers, and $65.0 \%$ for CCBs. The mean follow-up time was 1.6 years. Just over one third $(36.6 \%)$ of the cohort died during follow-up; cardiac-related causes accounted for $44.3 \%$ of the deaths. The combined CVD-endpoint occurred in $48.3 \%$ of the cohort. 
Table 2. AHRs for baseline factors associated with mortality and combined CVD-endpoint across exposure to any of the selected antihypertensive agents, time-dependent modeling of exposures ${ }^{1}$

\begin{tabular}{|c|c|c|c|c|}
\hline & \multicolumn{2}{|c|}{ All-cause mortality } & \multicolumn{2}{|c|}{ Combined CVD-endpoint } \\
\hline & AHR & $99 \% \mathrm{CI}$ & AHR & $99 \% \mathrm{CI}$ \\
\hline Age, per year & 1.029 & $1.027-1.031$ & 1.014 & $1.012-1.015$ \\
\hline Female sex & 0.979 & $0.939-1.021$ & 1.037 & $1.000-1.075$ \\
\hline \multicolumn{5}{|l|}{ Race/ethnicity } \\
\hline Caucasian & 1.0 & - & 1.0 & - \\
\hline African-American & 0.773 & $0.731-0.817$ & 0.856 & $0.813-0.901$ \\
\hline Hispanic & 0.673 & $0.631-0.718$ & 0.861 & $0.814-0.912$ \\
\hline Other & 0.570 & $0.520-0.625$ & 0.736 & $0.679-0.798$ \\
\hline \multicolumn{5}{|l|}{ BMI category } \\
\hline$<20$ & 1.258 & $1.182-1.338$ & 1.043 & $0.982-1.108$ \\
\hline $20-24.9$ & 1.0 & - & 1.0 & \\
\hline $25-29.9$ & 0.838 & $0.797-0.881$ & 0.950 & $0.909-0.992$ \\
\hline $30+$ & 0.744 & $0.707-0.782$ & 0.893 & $0.855-0.933$ \\
\hline Missing BMI & 0.888 & $0.750-1.051$ & 1.009 & $0.870-1.169$ \\
\hline Smoker & 1.156 & $1.070-1.249$ & 1.144 & $1.070-1.223$ \\
\hline Substance abuser & 1.193 & $1.056-1.348$ & 1.058 & $0.951-1.177$ \\
\hline Employed & 0.635 & $0.511-0.790$ & 0.940 & $0.821-1.077$ \\
\hline Inability to ambulate & 1.306 & $1.197-1.425$ & 0.969 & $0.888-1.057$ \\
\hline Inability to transfer & 1.208 & $1.058-1.380$ & 1.042 & $0.905-1.199$ \\
\hline \multicolumn{5}{|l|}{ Comorbidities } \\
\hline Diabetes & 1.198 & $1.141-1.258$ & 1.228 & $1.175-1.283$ \\
\hline Congestive heart failure & 1.231 & $1.183-1.282$ & 1.301 & $1.256-1.348$ \\
\hline Coronary artery disease & 1.102 & $1.054-1.153$ & 1.259 & $1.209-1.311$ \\
\hline Cerebrovascular accident & 1.174 & $1.112-1.239$ & 1.069 & $1.016-1.124$ \\
\hline Peripheral vascular disease & 1.066 & $1.013-1.121$ & 1.094 & $1.045-1.145$ \\
\hline Propensity score & 0.964 & $0.905-1.027$ & 0.994 & $0.940-1.052$ \\
\hline Self-care dialysis & 1.023 & $0.933-1.123$ & 0.923 & $0.853-1.000$ \\
\hline
\end{tabular}

${ }^{1}$ Drug effects shown separately (see suppl. table 3 ).

The distribution of cardiac hospitalizations consisted of CHF (53.2\%), CAD (20.1\%), CVA (14.9\%), and PVD (11.9\%). CVD deaths were mostly cardiac arrest (54.2\%) followed by CAD (21.4\%), CVA (9.8\%), arrhythmia (8.6\%), and cardiomyopathy (6.0\%).

\section{Statistical Models}

In the time-dependent model of combined drug class exposure (table 2), mortality was lower among non-Caucasians, persons with higher BMIs, and those who were employed. Conversely, smokers, substance abusers and persons who had impaired ability to ambulate or transfer had higher mortality risks. All selected comorbidities were associated with higher mortality, the strongest effects being seen in persons with CHF, diabetes, and prior CVA.

With respect to the time-dependent drug effects in the ACM model, we found a significant three-way interac- tion between the drug exposure measures, which we have depicted in the form of a three-dimensional graph (fig. 1). Blue-shaded areas indicate regions of benefit, with darker blue signaling higher benefit: red-shaded areas indicate regions of poor outcomes or increased risk for mortality, also with darker colors indicating the worst effects. Across the entirety of the space, persons who were actively on drug had lower hazards for mortality as compared to their peers. The degree of benefit, however, depended upon their cumulative exposure and the number of switches between on and off drug they had. For individuals who were currently on any of the agents, had only one switch, and had $90 \%$ of their weeks on an agent, their adjusted hazard ratio (AHR) was 0.560 (99\% CI 0.515-0.608). As one looks across the graph, one sees that an increasing number of switches was generally associated with less effectiveness shown by increasingly reddened areas, except for the cases in which the percent of weeks exposed was 
Fig. 1. Graphical representation of threeway interaction showing AHRs ACM for persons using compared to those not using selected antihypertensive agents in a given week. HR_ABCs_ACM = AHR for ACM based upon exposure to renin angiotensin system antagonists, $\beta$-adrenergic blockers, and/or calcium channel blockers (ABCs). Three-way interaction terms include (1) exposed compared to not exposed to selected antihypertensive in a given week, (2) proportion of prior weeks exposed (cumulative exposure \%), and (3) number of switches between exposed and not exposed weeks. Red depicts higher hazard ratios $(>1.0)$, while blue depicts lower hazard ratios $(<1.0)$.

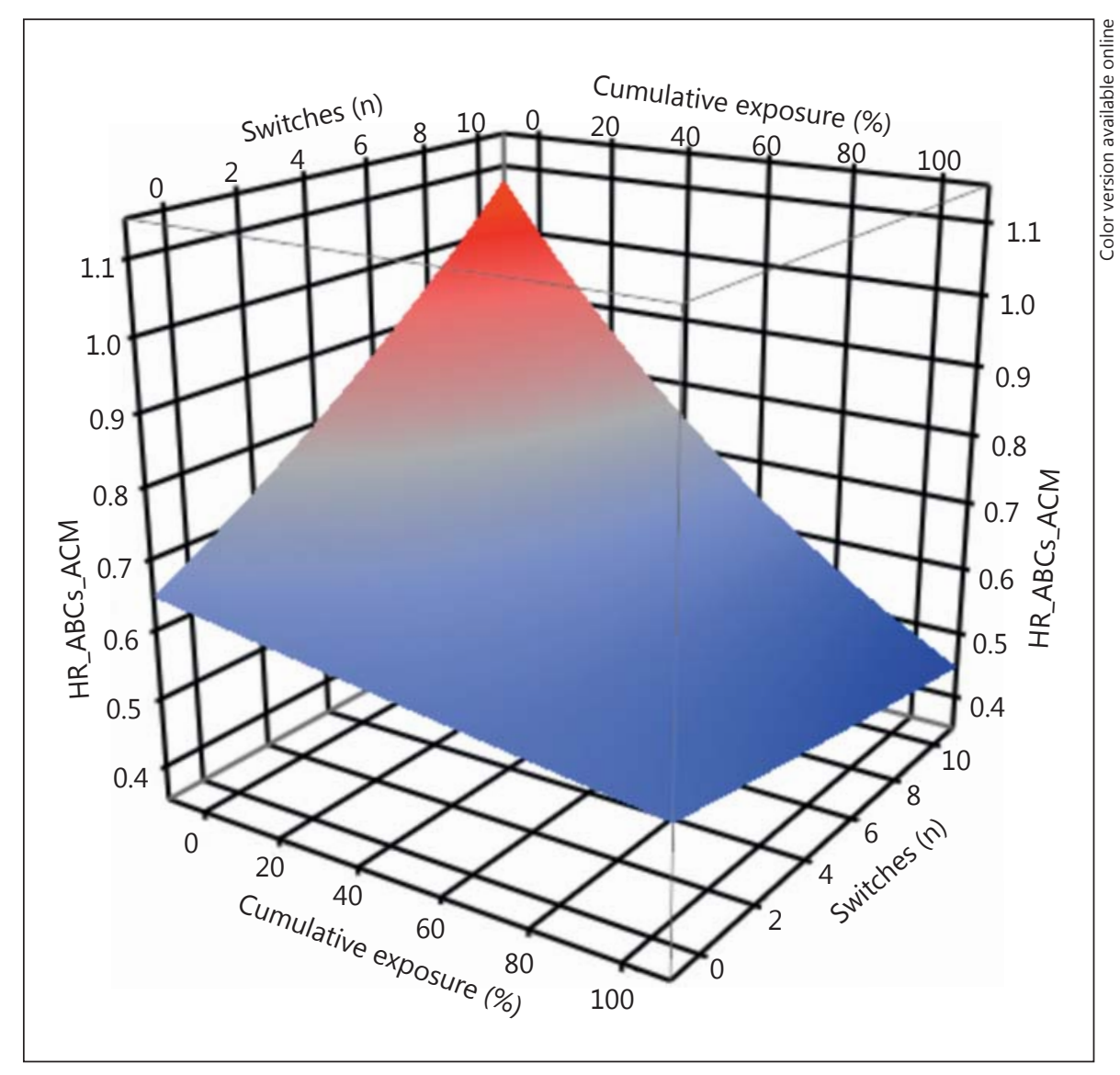

relatively high (i.e. $>50 \%$ ). For example, for persons who had drug exposure for $50 \%$ of their weeks of observation, AHRs rose from 0.614 to 0.686 as switches increased from 1 to a maximum of 10 . Moving from higher to lower cumulative exposure, there was evidence of reduced effectiveness across the graph, e.g. moving from blue to red. For instance, individuals who experienced five switches had AHRs that dropped from 0.766 at $10 \%$ exposure compared to 0.543 at $90 \%$ exposure. Overall, the use of any of the three drug classes appeared effective in reducing mortality. The inclusion of levothyroxine exposure was not statistically significant: this suggests that our modeling approach is not simply subject to the measurement of exposure over time (results for individual classes are provided in the online suppl. tables S2-S4).

In the combined CVD-endpoint model, similar results were seen for the various covariates (table 2). For instance, non-Caucasians and persons with higher BMIs still had lower risks for the outcomes, but the effect sizes were muted when compared to the mortality model. Smoking remained significant, but the other covariates were no longer significant. Diabetes, $\mathrm{CHF}$, and CAD were the three main comorbidities significantly associated with the combined CVD-endpoint.

The impact of time-dependent drug exposure on combined CVD-endpoint was captured by a two-way interaction between percent of preceding weeks with drug and being an active versus non-active user of the medication: the three-way interaction was not significant. In comparing active users (on) to non-active users (off), the hazard rates for a poorer outcome were higher when subjects had $40 \%$ or fewer of their prior weeks on drug ranging from 1.07 up to 1.18 (red shades in fig. 2). Since switches did not interact significantly, the resulting figure is a flat plane in three-dimensional space. When exposure exceeded $90 \%$ of their time, however, benefits accrued as evidenced by the AHR $=0.90$ (99\% CI $0.84-0.97)$ and as shaded in blue in figure 2. There was a second two-way interaction between percentage of weeks with medications and number of switches. Across the spectrum, higher percentages of weeks of cumulative drug exposure led to better outcomes as compared to lower percentages, 
Fig. 2. Graphical representation of twoway interaction showing AHRs for combined CVD-endpoint for persons using compared to those not using selected antihypertensive agents in a given week. HR CombinedCVDEndpoint $=$ AHRs for combined CVD-endpoint based upon exposure to renin angiotensin system antagonists, $\beta$-adrenergic blockers, and/or calcium channel blockers. Two-way interaction terms include (1) exposed compared to not exposed to selected antihypertensive in a given week and (2) proportion of prior weeks exposed (cumulative exposure \%). Switches were not significant but indicate the number of switches between exposed and not exposed weeks. Red depicts higher hazard ratios $(>1.0)$, while blue depicts lower hazard ratios $(<1.0)$.

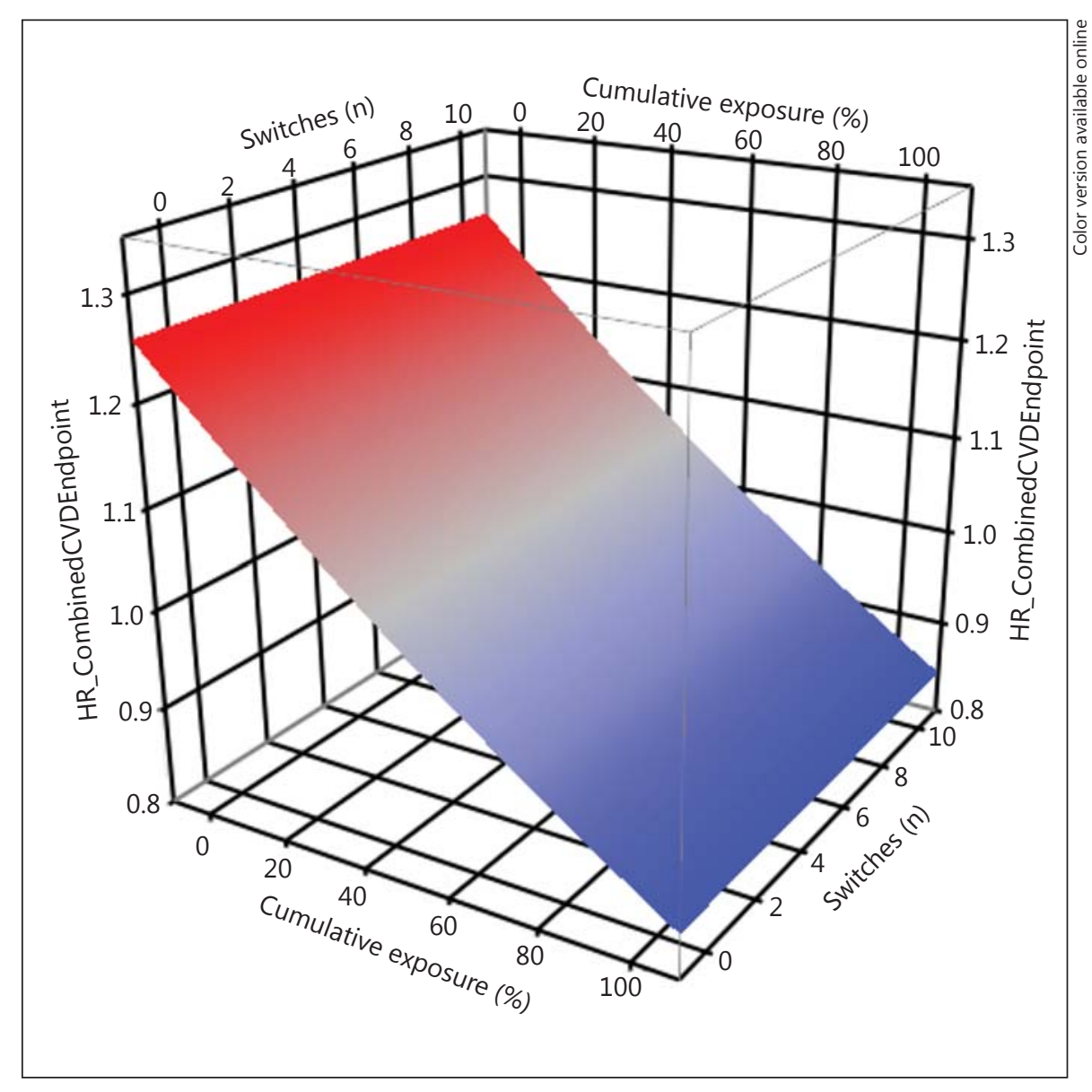

though the benefits of cumulative weekly exposure were reduced for persons who had more switches. As with the mortality model, the inclusion of levothyroxine exposure was not statistically significant (results for individual classes are provided in the online suppl. tables S2, S5-S7).

\section{Discussion}

Our research objective was to determine whether cardioprotective medications were used effectively to reduce mortality and morbidity in persons on maintenance dialysis with hypertension using novel time-dependent medication exposure measures and adjusting for confounding by indication using propensity scores. In general, exposure to cardioprotective medications was associated with lower ACM. The complexity of the time-dependent modeling precludes a single-point estimate for effectiveness for either outcome, but across a wide range of exposure and switching measures, most persons ac- tively and consistently taking one of these medications were less likely to die when compared to their peers not actively using these medications. The three time-dependent measures of drug exposure represent different components of medication use that warrant further discussion. First, we modeled each week during a person's window of observation as to whether or not, based upon refill patterns, they had access to the medication(s) during that week (the on/off variable). The advantage of this approach is that we captured exposure contemporaneously to the event, an important clinical concept. This week-byweek exposure is subject to some degree of misclassification since most prescriptions are dispensed on a monthly basis and the medication could have been discontinued before the week's indicator was turned to off. Persons who are off medication may be off their medication either by their own initiative (non-adherence) or because of a prescriber recommendation (medication discontinuation or hold). Holding of doses due to low blood pressure around the dialysis session may result in the accumula- 
tion of doses in the patient's home, but these would be smoothed out over a period of refills if patients refill when their bottles are nearly empty. Off time may also be higher for individuals who are near the end of life and foregoing further interventions, and as such, they would be more likely to die. We did explore this possible phenomenon but did not see a substantial decline in exposure during the last 12 weeks of life for those who died. Still, this may partially account for the larger effect size for ACM as compared to cardiovascular events.

The second component of the time-dependent exposure measure was the percentage of previous weeks for which the person had a prescription (on weeks). This measure essentially captures the cumulative level of exposure during the window of observation: however, it was only quantitatively different for different levels of the other two time-dependent measures. As was seen in our models, a higher percentage of time with drug exposure was associated with greater benefits, for mortality and, to a lesser extent, morbidity outcomes. From a clinical pharmacology perspective, one might expect that there would need to be a minimum period of exposure to produce therapeutic response. In fact, for the combined CVDendpoint model, benefits did not accrue until individuals had at least $90 \%$ of their time on the medication, and at lower percentages of exposure, the use of these drugs was associated with slightly increased risks. In the ACM model, at any level of drug exposure as captured by the percentage of weeks on drug, being on the drug was associated with reduced mortality, with higher benefits accrued at higher exposure levels. Given that the underlying comparison is with persons not on the drug in the given week, this measure arguably may be capturing a longer-term phenomenon.

The results of the time-dependent models also varied across the number of switches (week-by-week changes from on-to-off or off-to-on). In the ACM model, increasing numbers of switches were generally associated with lower degrees of benefit at lower percentages of weeks. At higher percentages of weeks with drug, the number of switches did not influence the effectiveness estimates quite as strongly. As with on/off status and the percentage of weeks with medication, switches collectively reflect patient and prescriber decisions. We argue that higher switch rates reflect more unstable patients, either clinically (e.g. hold/restart medications) and/or behaviorally (e.g. more non-adherent), and so apparent benefits might be lessened. In the case of the combined CVD-endpoint model, there were larger risk reductions in persons with fewer switches, consistent with the ACM model.
It is important to consider several limitations to this observational study. First, we did not have access to actual blood pressure measures and other potentially important confounders. We used propensity score adjustment in the outcome models to help balance measured confounders, but it is widely known that this approach is limited in its ability to account for unmeasured confounding. Our results reflect the use of these medications in clinical practice (e.g. assumes that clinicians are adjusting, holding, and discontinuing medications in response to contemporary changes in blood pressure) and, as such, should not be considered absolute measures of medication efficacy. Next, we relied on the CMS 2728 dialysis intake form to determine comorbidities, including hypertension [40]. This form has been shown to have good sensitivity and specificity for most but not all major comorbidities [41]. While patients may have acquired hypertension after the initiation of dialysis, it is unlikely that it would have resolved after starting it. While we were able to track medications over time, the presence of a claim for medication dispensation does not mean consumption. Those not actively on one of the medications may well have suffered an adverse event necessitating the termination of active treatment. We did not account for patients who may have voluntarily withdrawn dialysis and/or moved to hospice care. For these individuals, exposure based upon refills patterns may have been overstated, e.g. patients had medications they were no longer consuming. Although the data cover a time period preceding the implementation of the Medicare Part D drug benefit, the therapeutic options within the three drug classes have not changed substantively. The advantage of our slightly older data is that we have avoided misclassification biases that would result from USD 4 prescriptions and missing drug claims experienced during the coverage gap. Finally, we studied only dually eligible maintenance dialysis patients, which may decrease our ability to generalize findings to the US dialysis population as a whole.

We did not examine all classes of antihypertensives, e.g. diuretics and vasodilators. Our goal was to study antihypertensive medications with ostensible cardioprotective properties, and nearly $90 \%$ of the cohort had exposure to one of the classes during their follow-up period. This collective grouping of cardioprotective medications in the context of hypertension misses important opportunities for more direct analyses of specific patient risk groups. For instance, the recently published trial comparing atenolol to lisinopril in the context of HD patients with left ventricular hypertrophy and hypertension was terminated early due to significant differences in out- 
comes across the treatment arms [21]. We are actively pursuing within-class comparisons to identify potential targets for subsequent head-to-head trials.

Prior observational studies have suggested that cardioprotective medications offer mortality benefits for patients on maintenance dialysis [22-36], though these studies generally had less rigorous adjustments for potential selection bias and their data preceded widespread use of cardioprotective medications in maintenance dialysis patients. We developed and tested a novel set of three measures designed to characterize medication exposure over time. These measures collectively reflect current use, cumulative use, and stability of use over time. Based upon our results, current clinical practice with respect to cardioprotective medication use in maintenance dialysis patients is associated with reductions in mortality and morbidity. Using a new approach to capturing medication exposure over time, we demonstrated that effectiveness depends not only on having a drug available but is tempered by patients' patterns of drug use. These patterns likely reflect prescriber and patient behaviors, but generally support the continued use of cardioprotective medications in maintenance dialysis patients.

\section{Acknowledgements}

The research team is indebted to significant contributions by Purna Mukhopadhyay, PhD, and Qingjiang Hou, MS, for their substantive efforts early in the project at readying the files for analyses.

Funding for this study was provided by NIH (NIDDK) grants R01 DK080111 (T.I.S.) and K23 DK085378-01 (J.B.W.), by a National Kidney Foundation Young Investigator Award (J.B.W.), and by a Sandra A. Daugherty Foundation Grant (J.B.W.).

\section{Disclosure Statement}

The investigators report no conflicts of interest with the study and results as presented.

The data reported here have been supplied by the United States Renal Data System (DUA\#2007-10 \& 2009-19) and the Centers for Medicare \& Medicaid Services (DUA\#19707). The interpretation and reporting of these data are the responsibility of the author(s) and in no way should be seen as an official policy or interpretation of the US government.

\section{References}

$\checkmark 1$ Lopes AA, Bragg-Gresham JL, Ramirez SP, Andreucci VE, Akiba T, Saito A, et al: Prescription of antihypertensive agents to haemodialysis patients: time trends and associations with patient characteristics, country and survival in the DOPPS. Nephrol Dial Transplant 2009;24:2809-2816.

2 United States Renal Data System. USRDS 2006 Annual Data Report: Atlas of End-Stage Renal Disease in the United States. Bethesda, National Institutes of Health, National Institute of Diabetes and Digestive and Kidney Disease, 2006

- 3 Estacio RO, Jeffers BW, Hiatt WR, Biggerstaff SL, Gifford N, Schrier RW: The effect of nisoldipine as compared with enalapril on cardiovascular outcomes in patients with non-insulin-dependent diabetes and hypertension. $\mathrm{N}$ Engl J Med 1998;338:645-652.

-4 Metoprolol CR/XL Randomised Intervention Trial in Congestive Heart Failure (MERIT$\mathrm{HF}$ ): Effect of metoprolol CR/XL in chronic heart failure. Lancet 1999;353:2001-2007.

5 Yusuf S, Sleight P, Pogue J, Bosch J, Davies R, Dagenais G: Effects of an angiotensin-converting enzyme inhibitor, ramipril, on cardiovascular events in high-risk patients. The Heart Outcomes Prevention Evaluation Study Investigators. N Engl J Med 2000;342: 145-153.
6 Dargie HJ: Effect of carvedilol on outcome after myocardial infarction in patients with leftventricular dysfunction: the CAPRICORN randomised trial. Lancet 2001;357:1385-1390.

-7 Brenner BM, Cooper ME, de Zeeuw D, Keane WF, Mitch WE, Parving HH, et al: Effects of losartan on renal and cardiovascular outcomes in patients with type 2 diabetes and nephropathy. N Engl J Med 2001;345:861-869.

8 Kjeldsen SE, Dahlof B, Devereux RB, Julius S, Aurup P, Edelman J, et al: Effects of losartan on cardiovascular morbidity and mortality in patients with isolated systolic hypertension and left ventricular hypertrophy: a Losartan Intervention for Endpoint Reduction (LIFE) substudy. JAMA 2002;288:1491-1498.

$\checkmark 9$ Poole-Wilson PA, Swedberg K, Cleland JG, Di Lenarda A, Hanrath P, Komajda M, et al: Comparison of carvedilol and metoprolol on clinical outcomes in patients with chronic heart failure in the Carvedilol Or Metoprolol European Trial (COMET): randomised controlled trial. Lancet 2003;362:7-13.

10 McMurray JJ, Ostergren J, Swedberg K, Granger CB, Held P, Michelson EL, et al: Effects of candesartan in patients with chronic heart failure and reduced left-ventricular systolic function taking angiotensin-converting enzyme inhibitors: the CHARM-Added trial. Lancet 2003;362:767-771.
-11 Pfeffer MA, McMurray JJ, Velazquez EJ, Rouleau JL, Kober L, Maggioni AP, et al: Valsartan, captopril, or both in myocardial infarction complicated by heart failure, left ventricular dysfunction, or both. N Engl J Med 2003; 349:1893-1906.

12 Wassertheil-Smoller S, Psaty B, Greenland P, Oberman A, Kotchen T, Mouton C, et al: Association between cardiovascular outcomes and antihypertensive drug treatment in older women. JAMA 2004;292:28492859.

13 Demers C, McMurray JJ, Swedberg K, Pfeffer MA, Granger CB, Olofsson B, et al: Impact of candesartan on nonfatal myocardial infarction and cardiovascular death in patients with heart failure. JAMA 2005;294:17941798.

14 Dahlof B, Sever PS, Poulter NR, Wedel H, Beevers DG, Caulfield M, et al: Prevention of cardiovascular events with an antihypertensive regimen of amlodipine adding perindopril as required versus atenolol adding bendroflumethiazide as required, in the AngloScandinavian Cardiac Outcomes Trial-Blood Pressure Lowering Arm (ASCOT-BPLA): a multicentre randomised controlled trial. Lancet 2005;366:895-906. 
15 Jamerson K, Weber MA, Bakris GL, Dahlof B, Pitt B, Shi V, et al: Benazepril plus amlodipine or hydrochlorothiazide for hypertension in high-risk patients. N Engl J Med 2008;359: 2417-2428.

16 Cohn JN, Tognoni G: A randomized trial of the angiotensin-receptor blocker valsartan in chronic heart failure. N Engl J Med 2001;345: 1667-1675.

17 Fox KM: Efficacy of perindopril in reduction of cardiovascular events among patients with stable coronary artery disease: randomised, double-blind, placebo-controlled, multicentre trial (the EUROPA study). Lancet 2003 362:782-788.

18 Packer M, Coats AJ, Fowler MB, Katus HA, Krum H, Mohacsi P, et al: Effect of carvedilol on survival in severe chronic heart failure. $\mathrm{N}$ Engl J Med 2001;344:1651-1658.

$\checkmark 19$ Heerspink HJ, Ninomiya T, Zoungas S, de Zeeuw D, Grobbee DE, Jardine MJ, et al: Effect of lowering blood pressure on cardiovascular events and mortality in patients on dialysis: a systematic review and meta-analysis of randomised controlled trials. Lancet 2009; 373:1009-1015.

20 Agarwal R, Sinha AD: Cardiovascular protection with antihypertensive drugs in dialysis patients: systematic review and meta-analysis. Hypertension 2009;53:860-866.

-21 Agarwal R, Sinha AD, Pappas MK, Abraham TN, Tegegne GG: Hypertension in hemodialysis patients treated with atenolol or lisinopril: a randomized controlled trial. Nephrol Dial Transplant 2014;29:672-681.

22 Wetmore JB, Shireman TI: The ABCs of cardioprotection in dialysis patients: a systematic review. Am J Kidney Dis 2009;53:457466.

23 Abbott KC, Trespalacios FC, Agodoa LY, Taylor AJ, Bakris GL: Beta-blocker use in long-term dialysis patients: association with hospitalized heart failure and mortality. Arch Intern Med 2004;164:2465-2471.
4 Foley RN, Herzog CA, Collins AJ: Blood pressure and long-term mortality in United States hemodialysis patients: USRDS Waves 3 and 4 Study. Kidney Int 2002;62:1784-1790.

25 Griffith TF, Chua BS, Allen AS, Klassen PS, Reddan DN, Szczech LA: Characteristics of treated hypertension in incident hemodialysis and peritoneal dialysis patients. Am J Kidney Dis 2003;42:1260-1269.

26 Ishani A, Herzog CA, Collins AJ, Foley RN: Cardiac medications and their association with cardiovascular events in incident dialysis patients: cause or effect? Kidney Int 2004;65: 1017-1025.

27 Kestenbaum B, Gillen DL, Sherrard DJ, Seliger S, Ball A, Stehman-Breen C: Calcium channel blocker use and mortality among patients with end-stage renal disease. Kidney Int 2002;61:2157-2164.

28 Trespalacios FC, Taylor AJ, Agodoa LY, Abbott KC: Incident acute coronary syndromes in chronic dialysis patients in the United States. Kidney Int 2002;62:1799-1805.

29 Berger AK, Duval S, Krumholz HM: Aspirin, $\beta$-blocker, and angiotensin-converting enzyme inhibitor therapy in patients with endstage renal disease and an acute myocardial infarction. J Am Coll Cardiol 2003;42:201208.

30 Chow FY, Polkinghorne KR, Chadban SJ, At kins RC, Kerr PG: Cardiovascular risk in dialysis patients: a comparison of risk factors and cardioprotective therapy between 1996 and 2001. Nephrology (Carlton) 2003;8:177183.

-31 Efrati S, Zaidenstein R, Dishy V, Beberashvili I, Sharist M, Averbukh Z, et al: ACE inhibitors and survival of hemodialysis patients. Am J Kidney Dis 2002;40:1023-1029.

32 McCullough PA, Sandberg KR, Borzak S, Hudson MP, Garg M, Manley HJ: Benefits of aspirin and $\beta$-blockade after myocardial infarction in patients with chronic kidney disease. Am Heart J 2002;144:226-232.
33 Tepel M, Giet MV, Park A, Zidek W: Association of calcium channel blockers and mortality in haemodialysis patients. Clin Sci (Lond) 2002; 103:511-515.

34 Winkelmayer WC, Charytan DM, Levin R, Avorn J: Poor short-term survival and low use of cardiovascular medications in elderly dialysis patients after acute myocardial infarction. Am J Kidney Dis 2006;47:301-308.

35 Trespalacios FC, Taylor AJ, Agodoa LY, Bakris GL, Abbott KC: Heart failure as a cause for hospitalization in chronic dialysis patients. Am J Kidney Dis 2003;41:1267-1277.

36 Boger CA, Gotz AK, Kruger B, Hosl M, Schmitz G, Riegger GA, et al: Effect of genetic variation on therapy with angiotensin-converting enzyme inhibitors or angiotensin receptor blockers in dialysis patients. Eur J Med Res 2005;10:161-168.

37 Rigler SK, Wetmore JB, Mahnken JD, Dong L, Ellerbeck EF, Shireman TI: Impact of a modified data capture period on Liu comorbidity index scores in Medicare enrollees initiating chronic dialysis. BMC Nephrol 2013; $14: 51$

38 Liu J, Huang Z, Gilbertson DT, Foley RN, Collins AJ: An improved comorbidity index for outcome analyses among dialysis patients. Kidney Int 2010;77:141-151.

39 Schneeweiss S, Patrick AR, Sturmer T, Brookhart MA, Avorn J, Maclure M, et al: Increasing levels of restriction in pharmacoepidemiologic database studies of elderly and comparison with randomized trial results. Med Care 2007;45(suppl 2):S131-S142.

40 Eggers PW: CMS 2728: what good is it? Clin J Am Soc Nephrol 2010;5:1908-1909.

41 Longenecker JC, Coresh J, Klag MJ, Levey AS, Martin AA, Fink NE, et al: Validation of comorbid conditions on the end-stage renal disease medical evidence report: the CHOICE study. Choices for Healthy Outcomes in Caring for ESRD. J Am Soc Nephrol 2000;11: 520-529. 